\title{
Serum Apolipoprotein A-I Combined With C-Reactive Protein Serves As A Novel Prognostic Stratification System For Colorectal Cancer
}

This article was published in the following Dove Press journal: Cancer Management and Research

Juan Ye, ${ }^{1-3, *}$ Qiu-yun Luo, ${ }^{1, *}$ Xue-ping Wang, ${ }^{4}$ Zhenyi Liu, ${ }^{5}$ Mei-xian Chen, ${ }^{6}$ Hao Huang, ${ }^{7}$ Lin Zhang ${ }^{1,4}$

'Department of Experimental Research, State Key Laboratory of Oncology in South China, Collaborative Innovation Center for Cancer Medicine, Sun Yat-Sen University Cancer Center, Guangzhou, Guangdong, People's Republic of China; ${ }^{2}$ Department of Infectious Diseases, The Fifth Affiliated Hospital of Sun Yat-Sen University, Zhuhai, Guangdong, People's Republic of China;

${ }^{3}$ Department of Oncology, Affiliated Hospital of Zunyi Medical University, Zunyi, Guizhou, People's Republic of China; ${ }^{4}$ Department of Clinical Laboratory, Sun YatSen University Cancer Center, Guangzhou, Guangdong, People's Republic of China;

${ }^{5}$ Department of Blood Transfusion, Peking University Shenzhen Hospital, Shenzhen, Guangdong, People's Republic of China; ${ }^{6}$ Department of Hepatobiliary Oncology, Sun Yat-Sen University Cancer Center, Guangzhou, Guangdong, People's Republic of China; ${ }^{7}$ Department of Laboratory Science, First Affiliated Hospital of Sun YatSen University, Guangzhou, Guangdong, People's Republic of China

*These authors contributed equally to this work

Correspondence: Lin Zhang

Department of Clinical Laboratory, Sun

Yat-Sen University Cancer Center, 65I

Dongfeng Road East, Guangzhou 510060,

People's Republic of China

$\mathrm{Tel} / \mathrm{Fax}+862087342285$

Email zhanglin@sysucc.org.cn

Hao Huang

Department of Laboratory Science, First

Affiliated Hospital of Sun Yat-Sen University,

58 Zhongshan Second Road, Guangzhou

510080 , People's Republic of China

$\mathrm{Tel} / \mathrm{Fax}+862087330808$

Email836271002@qq.com
Background and objective: Noninvasive prognostic tools for colorectal cancer (CRC) are urgently needed. This study was designed to investigate the prognostic value of preoperative serum lipid and lipoprotein concentrations (including ApoA-I, Apo-B, HDL-C, LDL-C, TC and TG) and CRP levels retrospectively in CRC patients.

Methods: Preoperative serum lipid and lipoprotein concentrations (including ApoA-I, ApoB, HDL-C, LDL-C, TC and TG) and CRP levels were analyzed retrospectively in 250 patients with CRC. The prognostic significance of these indexes was determined by univariate and multivariate Cox hazard models.

Results: CRC patients with higher levels of ApoA-I and HDL-C and lower levels of CRP had significantly longer overall survival (OS, log rank test, $p<0.05)$. Based on univariate analysis, ApoA-I levels $(p=0.002)$, CRP levels $(p=0.007)$, HDL-C levels $(p=0.005)$, pT classification $(p=0.005), \mathrm{pN}$ classification $(p<0.001), \mathrm{pM}$ classification $(p<0.001)$ and pTNM stage $(p<0.001)$ were significantly associated with OS. Multivariate Cox proportional hazards regression analysis indicated that ApoA-I levels (HR: 1.52, $p=0.023$ ), CRP levels (HR: 1.85, $p=0.035$ ) and pTNM stage (HR: $2.53, p<0.001$ ) were independent predictors of CRC survival. The included patients were then stratified into three tiers based on the ApoA-I and CRP levels. In the whole cohort, the OS and disease-free survival differed significantly between the lowrisk (ApoA-I $\geq 1.08 \mathrm{mg} / \mathrm{dL}$ and $\mathrm{CRP}<3.04 \mathrm{mg} / \mathrm{dL}$ ), medium-risk (ApoA-I $\geq 1.08 \mathrm{mg} / \mathrm{dL}$ or $\mathrm{CRP}<3.04 \mathrm{mg} / \mathrm{dL}$ ), and high-risk (ApoA-I $<1.08 \mathrm{mg} / \mathrm{dL}$ and $\mathrm{CRP} \geq 3.04 \mathrm{mg} / \mathrm{dL}$ ) groups $(p=0.001$ and $p=0.004)$.

Conclusion: Decreased levels of ApoA-I and HDL-C and increased levels of CRP were predictive of poor prognosis among patients with CRC. In addition, the combination of ApoA-I and CRP can serve as a novel prognostic stratification system for more accurate clinical staging of CRC.

Keywords: apolipoprotein A-I, CRP, CRC, prognosis

\section{Introduction}

Colorectal cancer (CRC) is one of the most common forms of cancer worldwide. CRC accounts for $8 \%$ of all cancer-related deaths, representing the fourth most common cause of death from cancer. ${ }^{1}$ Moreover, the incidence of CRC is rapidly increasing, and the substantial interest in identifying potential prognostic factors for CRC has kept pace with the increasing incidence of this disease. Although many serum biomarkers have been reported to predict the survival of CRC patients, the sensitivity and specificity of these markers are limited; thus, these markers are not 
entirely reliable. ${ }^{2-4}$ Therefore, it is necessary to identify accurate biomarkers for CRC to improve the clinical outcome of patients.

Lipids are composed of fats (triglyceride, TG) and lipids (phospholipid, cholesterol and cholesterol ester). Lipid metabolism involves the process of lipid synthesis, storage and degradation. ${ }^{5}$ Abnormal lipid metabolism has been shown to be closely related to tumor progression in several cancer types and under inflammatory conditions. ${ }^{6,7}$ In rapidly proliferating cancer cells, cholesterol synthesis is enhanced because excess cholesterol is needed to maintain a high rate of proliferation, and the requirement of metabolic intermediates for macromolecule production is overwhelming. ${ }^{8}$ Accordingly, cancer cells coordinate the activation of lipid anabolic metabolism and the corresponding signaling networks for membrane formation, energy storage, the production of signaling molecules, and as an important energy source to generate ATP via fatty acid oxidation under energy-deficient conditions. ${ }^{5,9}$ Evidence for the activation of lipid metabolism in tumor cells can be produced by quantifying the products of lipid metabolism, such as apolipoprotein A-I (ApoA-I), in the serum from some cancer patients. ${ }^{10-12}$

ApoA-I is the major protein component of high-density lipoprotein-cholesterol (HDL-C), ${ }^{13,14}$ which can also function as an enzyme cofactor, receptor ligand, and lipid carrier in the regulation of lipoprotein metabolism. ${ }^{7,15,16}$ Many studies have shown that ApoA-I has atheroprotective functions, including anti-inflammatory, antiapoptotic and antioxidant activities. ${ }^{17-19}$ Chronic inflammation, oxidative stress, lipids, and cholesterol have been associated with tumorigenesis. ${ }^{20-22}$ Given the beneficial effects of ApoA-I against these atherogenic processes, it was somewhat intuitive to hypothesize that ApoA-I may also be protective against cancer. ApoA-I may suppress tumor growth and metastasis primarily via the modulation of innate and adaptive immune responses. ${ }^{23}$ In the past ten years, a correlation of ApoA-I levels with disease risk has been observed for many cancer types. High levels of ApoA-I prior to chemotherapy were correlated with better overall survival in ovarian cancer $(\mathrm{OC})^{24,25}$ and nasopharyngeal carcinoma (NPC). ${ }^{26}$ Lower serum ApoA-I levels were associated with higher risks of breast cancer ${ }^{6}$ and pancreatic cancer. ${ }^{12}$ It has been reported that the plasma concentrations of HDL-C and ApoA-I were inversely associated with the risk of colon cancer. ${ }^{27}$ However, the prognostic value of serum ApoA-I levels for the overall survival of patients with CRC remains unclear.
In the present study, we retrospectively investigated the prognostic value of ApoA-I for overall survival among patients with CRC and then evaluated the relationship between ApoA-I levels and clinical characteristics. In addition, high levels of C-reactive protein (CRP), as well as inflammatory cytokines IL-1A, IL-1B and IL-8, were previously reported as predictive of survival in a much larger cohort of CRC patients. ${ }^{28-30}$ Therefore, we combined ApoA-I with CRP levels to serve as a prognostic stratification system for CRC to more accurately delineate meaningful prognostic subsets of the study population.

\section{Materials And Methods}

\section{Patient Selection}

A retrospective review was conducted by analyzing data on 250 patients (age range, 26-85 years; median age, 58 years) who underwent surgery at Sun Yat-Sen University Cancer Center (SYSUCC; Guangzhou, China) as a primary treatment for CRC between January 2008 and December 2010. All patients met the following eligibility criteria: 1) pathologically confirmed diagnosis of CRC with no previous or coexisting cancer; 2) no therapy prior to serum collection; 3) no concomitant disease that has been associated with increased serum lipid levels (i.e. diabetes, hyperlipidemia, or metabolic syndrome); and 4) no hormone replacement therapy or use of any drugs known to affect lipid metabolism.

The experiment was approved by the Institutional Research Ethics Committee of SYSUCC, Guangzhou, China, and written informed consent was obtained from each patient for the use of their biological samples for genetic analysis. This study was conducted in accordance with the Declaration of Helsinki.

Detailed clinical and pathological characteristics, including demographic variables, smoking history, alcohol history, pathological tumor node metastasis (pTNM) stage and overall survival, were available for all patients. Overall survival, defined as the time from surgery to death or the most recent follow-up, whichever occurred first, was used as a measure of patient prognosis. At the latest follow-up, 68 deaths occurred among the 250 patients examined.

\section{Laboratory Measurements}

The serum levels of ApoA-I, ApoB, CRP, HDL-C, lowdensity lipoprotein cholesterol (LDL-C), total cholesterol (TC), and triglycerides (TG) were collected from the 
preoperative medical records of the patients studied. Peripheral blood samples drawn from patients less than 2 weeks prior to surgery were measured using a Hitachi 7600-020 automatic biochemical analyzer (Hitachi Co., Japan) in the clinical laboratory of the SYSUCC. The TC level in whole serum samples was measured using the CHODPAP method; HDL-C and LDL-C were detected via the selective elimination method (direct method) and the selective protection method, respectively. Serum TG levels were measured using the GPO-PAP method. ApoAI and Apo-B levels were measured via immunoturbidimetry. All test kits were provided by Wako Pure Chemical Industries, Japan. The serum level of CRP was determined via latex immunological transmission turbidimetry, and the associated assay kits were provided by Sekisui Medical Co., Ltd., Japan.

The results are reported as either mean or median values, depending on the type of distribution. Then, the variables (ApoA-I, HDL-C, Apo-B, TG, TC and CRP) were categorized using median values as cut-off points, except that the cut-off point for LDL-C was based on the mean \pm SD. Therefore, an ApoA-I level of $1.08 \mathrm{~g} / \mathrm{L}$, an HDL-C level of $1.18 \mathrm{mmol} / \mathrm{L}$, a TG level of $1.29 \mathrm{mg} / \mathrm{dL}$, a cholesterol level of $5.08 \mathrm{mg} / \mathrm{dL}$, an LDL-C level of $3.18 \mathrm{mg} / \mathrm{dL}$, an Apo-B level of $0.97 \mathrm{mg} / \mathrm{dL}$ and a CRP level of $3.04 \mathrm{mg} / \mathrm{L}$ were selected as the cut-off points for the survival analysis. The interrelationships of clinicopathologic features, serum levels of ApoA-I, ApoB, CRP, HDL-C, LDL-C, TC and TG and the cancer prognosis were analyzed. Each patient returned to a specified division of SYSUCC on a semiannual basis for clinical follow-up visits, and follow-up information was recorded until death or at a maximum of 82 months (median: 45 months, range: 0-82 months) at the time of study completion (March 2016).

\section{Statistical Analysis}

Statistical analysis was performed using IBM SPSS version 20.0 for Windows (IBM Co., New York, NY). All reported $P$ values were two-sided, and $P$ values $<0.05$ were considered statistically significant. The correlations between lipid profiles and clinical characteristics were assessed using the Mann-Whitney $U$-test, the associations of LDL-C levels with clinical characteristics were evaluated using independent t-tests, and the analysis of TNM stage was conducted with the Kruskal-Wallis test. The chisquare test $\left(\chi^{2}\right.$ test) was also conducted to assess the association between the ApoA-I levels and the observed clinical characteristics of the CRC patients. Survival curves were plotted via the Kaplan-Meier method, and the log rank test was used to compare the differences in survival curves between groups and, the Cox proportional hazard regression model was employed to determine hazard ratio estimates. All variables shown to be significant based on the univariate analysis were then evaluated in a multivariate Cox proportional hazards model to identify the independent variables that were predictive of CRC patient survival.

\section{Results}

\section{Lipid Profiles And Their Correlations With Clinical Characteristics}

The relationships between the lipid profiles and the clinical characteristics of the patients are summarized in Table 1. Specifically, the patients who were female, had no alcohol history or metastasis and were classified as T1-T3 exhibited significantly higher ApoA-I levels ( $p=0.001, p=0.017$, $p=0.01$, and $p=0.001$, respectively). Additionally, the HDL-C level was significantly correlated with gender, tobacco history, alcohol history, pT classification, and metastasis $(p=0.001, p=0.05, \quad p=0.043, p=0.027$, and $p=0.047$, respectively). Furthermore, patients aged 58 years exhibited higher ApoB levels $(p=0.044)$. In addition, elevated TC levels were associated with gender and tobacco history ( $p=0.003$ and $p=0.036$ ), and patients classified as T4 and M1 exhibited higher CRP levels ( $p=0.001$ and $p=0.026$, respectively). However, none of the clinical characteristics was significantly associated with the LDL-C level. (Table 1)

\section{Kaplan-Meier Survival Analysis Of The Levels Of Independent Predictors In CRC}

To evaluate the prognostic value of pretreatment ApoA-I levels in CRC patients, the associations of clinical characteristics (including age, gender, tobacco history, alcohol history, pT classification, $\mathrm{pN}$ status, $\mathrm{pM}$ status, pTNM status, CRP levels and lipid levels) with overall survival were evaluated via univariate and multivariate analyses. As shown in Table 2, the univariate analysis revealed that ApoA-I levels [hazard ratio (HR): 0.47, $p=0.002$ ], HDL-C levels (HR: 0.50, $p=0.005$ ), CRP levels (HR: 1.95, $p=0.007$ ), pT classification (T1-3 vs T4, HR: 1.76 , $p=0.023), \mathrm{pN}$ classification (N1-N3 vs N0, HR: 3.48 , $p<0.001$ ), pM status (Yes vs No, HR: 6.46, $p<0.001$ ), and pTNM status (I vs II vs III vs IV, HR: 2.56, $p<0.001)$ were significantly associated with overall survival. Next, multivariate analysis was used to further determine whether these seven factors served as independent 
Table I Main Clinical Characteristics Of Patients Group According To Lipid Profile And CRP Levels (Median And Range)

\begin{tabular}{|c|c|c|c|c|c|c|c|c|}
\hline Variable & $\mathbf{N}$ & ApoA-I & HDL-C & LDL-C & ApoB & TG & TC & CRP \\
\hline $\begin{array}{l}\text { Age } \\
\qquad 58 \\
\geq 58\end{array}$ & $\begin{array}{l}119 \\
131\end{array}$ & $\begin{array}{l}1.04 \\
(0.46-1.85) \\
1.1 \\
(0.43-2.56) \\
P=0.29\end{array}$ & $\begin{array}{l}1.11 \\
(0.23-1.95) \\
1.13 \\
(0.28-6.94) \\
P=0.597\end{array}$ & $\begin{array}{l}3.03 \\
(1.66-5.56) \\
3.16 \\
(0.93-6.5 I) \\
P=0.16 I\end{array}$ & $\begin{array}{l}0.95 \\
(0.52-1.58) \\
1.04 \\
(0.47-41.37) \\
P=0.044\end{array}$ & $\begin{array}{l}1.29 \\
(0.58-7.47) \\
1.27 \\
(0.53-5.26) \\
P=0.956\end{array}$ & $\begin{array}{l}4.97 \\
(I .2 I-7.55) \\
5.22 \\
(0.7 I-7.8 I) \\
P=0.097\end{array}$ & $\begin{array}{l}2.57 \\
(0.09-127.09) \\
2.98 \\
(0.21-128.32) \\
P=0.845\end{array}$ \\
\hline $\begin{array}{l}\text { Gender } \\
\text { Male } \\
\text { Female }\end{array}$ & $\begin{array}{l}144 \\
106\end{array}$ & $\begin{array}{l}1.03 \\
(0.43-2.56) \\
1.14 \\
(0.55-2.01) \\
P=0.001\end{array}$ & $\begin{array}{l}1.07 \\
(0.23-6.94) \\
1.22 \\
(0.28-2.28) \\
P=0.001\end{array}$ & $\begin{array}{l}3.08 \\
(0.93-6.5 I) \\
3.19 \\
(I .72-5.56) \\
P=0.198\end{array}$ & $\begin{array}{l}0.96 \\
(0.47-3.10) \\
1.03 \\
(0.57-41.37) \\
P=0.080\end{array}$ & $\begin{array}{l}1.28 \\
(0.53-7.47) \\
1.27 \\
(0.55-4.47) \\
P=0.765\end{array}$ & $\begin{array}{l}4.97 \\
(0.7 I-7.63) \\
5.42 \\
(3.2 I-7.8 I) \\
P=0.003\end{array}$ & $\begin{array}{l}2.58 \\
(0.09-128.32) \\
3.07 \\
(0.26-127.09) \\
P=0.235\end{array}$ \\
\hline $\begin{array}{l}\text { Tobacco history } \\
\text { No } \\
\text { Yes }\end{array}$ & $\begin{array}{l}180 \\
70\end{array}$ & $\begin{array}{l}1.07 \\
(0.46-2.01) \\
1.04 \\
(0.43-2.56) \\
P=0.078\end{array}$ & $\begin{array}{l}1.13 \\
(0.48-2.28) \\
1.11 \\
(0.23-6.94) \\
P=0.050\end{array}$ & $\begin{array}{l}3.14 \\
(I .22-6.5 I) \\
3.08 \\
(0.93-5.56) \\
P=0.472\end{array}$ & $\begin{array}{l}I \\
(0.52-4 I .37) \\
0.96 \\
(0.47-3.10) \\
P=0.784\end{array}$ & $\begin{array}{l}1.32 \\
(0.53-7.47) \\
1.2 \\
(0.62-4.43) \\
P=0.349\end{array}$ & $\begin{array}{l}5.17 \\
(0.7 I-7.8 I) \\
4.9 I \\
(I .21-7.63) \\
P=0.036\end{array}$ & $\begin{array}{l}2.58 \\
(0.09-128.32) \\
3.1 \\
(0.25-70.56) \\
P=0.683\end{array}$ \\
\hline $\begin{array}{l}\text { Alcohol history } \\
\text { No } \\
\text { Yes }\end{array}$ & $\begin{array}{l}217 \\
32\end{array}$ & $\begin{array}{l}1.08 \\
(0.43-2.56) \\
1.01 \\
(0.69-1.36) \\
P=0.017\end{array}$ & $\begin{array}{l}1.13 \\
(0.23-6.94) \\
1.08 \\
(0.43-1.42) \\
P=0.043\end{array}$ & $\begin{array}{l}3.13 \\
(I .22-6.5 I) \\
3.05 \\
(0.93-4.18) \\
P=0.141\end{array}$ & $\begin{array}{l}0.99 \\
(0.52-41.37) \\
0.97 \\
(0.47-1.29) \\
P=0.275\end{array}$ & $\begin{array}{l}1.24 \\
(0.53-7.47) \\
1.48 \\
(0.65-5.11) \\
P=0.259\end{array}$ & $\begin{array}{l}5.15 \\
(0.7 I-7.8 I) \\
4.9 \\
(2.63-6.67) \\
P=0.098\end{array}$ & $\begin{array}{l}2.76 \\
(0.09-128.32) \\
2.97 \\
(0.32-39.60) \\
P=0.536\end{array}$ \\
\hline $\begin{array}{l}\text { pT Classification } \\
\text { TI-T3 } \\
\text { T4 }\end{array}$ & $\begin{array}{l}127 \\
123\end{array}$ & $\begin{array}{l}1.13 \\
(0.43-2.56)\end{array}$ & $\begin{array}{l}1.17 \\
(0.25-6.94)\end{array}$ & $\begin{array}{l}3.09 \\
(1.56-5.77)\end{array}$ & $\begin{array}{l}1.01 \\
(0.52-3.10)\end{array}$ & $\begin{array}{l}1.36 \\
(0.59-7.47)\end{array}$ & $\begin{array}{l}5.18 \\
(1.21-7.81)\end{array}$ & $\begin{array}{l}1.9 \\
(0.19-70.56)\end{array}$ \\
\hline $\begin{array}{l}\text { pT Classification } \\
\text { TI-T3 } \\
\text { T4 }\end{array}$ & $\begin{array}{l}127 \\
123\end{array}$ & $\begin{array}{l}1.13 \\
(0.43-2.56) \\
1.04 \\
(0.46-1.85) \\
P=0.001\end{array}$ & $\begin{array}{l}1.17 \\
(0.25-6.94) \\
1.11 \\
(0.23-1.95) \\
P=0.027\end{array}$ & $\begin{array}{l}3.09 \\
(1.56-5.77) \\
3.10 \\
(0.93-6.5 I) \\
P=0.736\end{array}$ & $\begin{array}{l}I .01 \\
(0.52-3.10) \\
0.97 \\
(0.47-41.37) \\
P=0.79\end{array}$ & $\begin{array}{l}1.36 \\
(0.59-7.47) \\
1.22 \\
(0.53-5.52) \\
P=0.226\end{array}$ & $\begin{array}{l}5.18 \\
(1.21-7.8 I) \\
4.93 \\
(0.7 I-7.56) \\
P=0.233\end{array}$ & $\begin{array}{l}1.9 \\
(0.19-70.56) \\
4.74 \\
(0.09-128.32) \\
P=0.001\end{array}$ \\
\hline $\begin{array}{l}\text { pN Classification } \\
\text { No } \\
\text { NI-N3 }\end{array}$ & $\begin{array}{l}116 \\
110\end{array}$ & $\begin{array}{l}1.08 \\
(0.60-2.01) \\
1.06\end{array}$ & $\begin{array}{l}1.13 \\
(0.67-2.28) \\
1.1\end{array}$ & $\begin{array}{l}3.19 \\
(1.22-6.5 I) \\
3.07\end{array}$ & $\begin{array}{l}1.01 \\
(0.57-1.80) \\
0.96\end{array}$ & $\begin{array}{l}1.22 \\
(0.53-7.47) \\
1.37\end{array}$ & $\begin{array}{l}5.16 \\
(1.21-7.81) \\
4.99\end{array}$ & $\begin{array}{l}3.06 \\
(0.19-128.32) \\
2.55\end{array}$ \\
\hline $\begin{array}{l}\text { pMetastasis } \\
\text { No } \\
\text { Yes }\end{array}$ & 173 & $\begin{array}{l}1.09 \\
(0.51-2.56) \\
1 \\
(0.46-1.85) \\
P=0.010\end{array}$ & $\begin{array}{l}1.13 \\
(0.23-6.94) \\
1.08 \\
(0.48-1.95) \\
P=0.047\end{array}$ & $\begin{array}{l}3.12 \\
(0.93-5.56) \\
3.12 \\
(1.92-6.5 I) \\
P=0.928\end{array}$ & $\begin{array}{l}1.01 \\
(0.47-41.37) \\
0.95 \\
(0.62-1.80) \\
P=0.596\end{array}$ & $\begin{array}{l}1.23 \\
(0.53-7.47) \\
1.41 \\
(0.59-2.86) \\
P=0.653\end{array}$ & $\begin{array}{l}5.1 \\
(1.21-7.8 I) \\
5.1 \\
(0.71-7.56) \\
P=0.429\end{array}$ & $\begin{array}{l}2.45 \\
(0.09-127.09) \\
4.65 \\
(0.31-128.32) \\
P=0.026\end{array}$ \\
\hline pTNM Stage & $\begin{array}{l}\& \\
30\end{array}$ & $\begin{array}{l}1.11 \\
(0.43-1.75)\end{array}$ & $\begin{array}{l}1.18 \\
(0.25-1.94)\end{array}$ & $\begin{array}{l}3.07 \\
(1.74-5.56)\end{array}$ & $\begin{array}{l}0.97 \\
(0.59-1.58)\end{array}$ & $\begin{array}{l}1.26 \\
(0.65-3.95)\end{array}$ & $\begin{array}{l}4.92 \\
(3.21-7.55)\end{array}$ & $\begin{array}{l}1.95 \\
(0.19-55.74)\end{array}$ \\
\hline
\end{tabular}

(Continued) 
Table I (Continued).

\begin{tabular}{|c|c|c|c|c|c|c|c|c|}
\hline Variable & $\mathbf{N}$ & ApoA-I & HDL-C & LDL-C & ApoB & TG & TC & CRP \\
\hline II & 77 & $\begin{array}{l}I .13 \\
(0.09-2.01)\end{array}$ & $\begin{array}{l}\text { I.18 } \\
(0.43-2.28)\end{array}$ & $\begin{array}{l}3.22 \\
(1.22-5.5 \mathrm{I})\end{array}$ & $\begin{array}{l}1.03 \\
(0.57-1.66)\end{array}$ & $\begin{array}{l}\text { I.23 } \\
(0.53-7.47)\end{array}$ & $\begin{array}{l}5.2 \\
(I .2 I-7.8 I)\end{array}$ & $\begin{array}{l}2.76 \\
(0.37-81.55)\end{array}$ \\
\hline III & 85 & $\begin{array}{l}1.09 \\
(0.51-2.56)\end{array}$ & $\begin{array}{l}1.15 \\
(0.23-6.94)\end{array}$ & $\begin{array}{l}3.05 \\
(0.93-5.77)\end{array}$ & $\begin{array}{l}0.99 \\
(0.47-41.37)\end{array}$ & $\begin{array}{l}1.27 \\
(0.58-5.52)\end{array}$ & $\begin{array}{l}5.04 \\
(1.89-7.29)\end{array}$ & $\begin{array}{l}3 \\
(0.09-127.09)\end{array}$ \\
\hline IV & 58 & $\begin{array}{l}1.03 \\
(0.46-1.85) \\
P=0.116\end{array}$ & $\begin{array}{l}1.06 \\
(0.48-1.95) \\
P=0.19\end{array}$ & $\begin{array}{l}3.06 \\
(I .7 I-6.5 I) \\
P=0.654\end{array}$ & $\begin{array}{l}0.94 \\
(0.62-\mid .80) \\
P=0.68 \mid\end{array}$ & $\begin{array}{l}I .4 I \\
(0.59-2.86) \\
P=1.000\end{array}$ & $\begin{array}{l}4.79 \\
(0.7 I-7.56) \\
P=0.393\end{array}$ & $\begin{array}{l}4.46 \\
(0.31-128.32) \\
P=0.324\end{array}$ \\
\hline
\end{tabular}

Notes: Mann-Whitney U-test was used in Table I, except that the associations of LDL-C levels with clinical characteristics were evaluated using independent $t$-tests, and the analysis of TNM stage was Kruskal-Wallis test. \&TNM denoted tumor-node-metastasis. Bold italics indicate significant differences $(p<0.05)$.

prognostic factors for survival. Concerning the influence of statistical collinearity, the multivariate model did not include $\mathrm{pT}$ status, $\mathrm{pN}$ status, and $\mathrm{pM}$ status. The multivariate analysis showed that ApoA-I levels (HR: 1.52, $p=0.023$ ), CRP levels (HR: 1.85, $p=0.035$ ), and pTNM stage (HR: 2.53, $p<0.001$ ) were significant independent predictors of CRC patient survival. (Table 2)

Furthermore, the Kaplan-Meier method was used to plot survival curves and to estimate the prognostic significance of ApoA-I and the other six independent predictors of overall survival and disease-free survival for CRC patients. As shown in Figure 1, the log rank test revealed that higher CRP levels $(p=0.005)$, lower ApoA-I and HDL-C levels ( $p=0.006$ and $p=0.010$, respectively), pT classification $(p=0.020), \mathrm{pN}$ classification $(p=0.001), \mathrm{pM}$ status $(p=0.001)$ and pTNM stage $(p=0.001)$ were predictive of poorer overall survival among patients with CRC. In addition, the elevation of ApoA-I $(p=0.007)$, CRP $(p=0.048)$, and HDL-C $(p=0.016)$ levels, $\mathrm{pT}$ classification $(p=0.021), \mathrm{pN}$ classification $(p=0.001), \mathrm{pM}$ classification $(p=0.001)$ and $\mathrm{pTNM}$ stage $(p=0.001)$ portended poorer disease-free survival for CRC patients. (Figure 2)

\section{Relationship Between ApoA-I Levels And Clinical Characteristics In 250 Patients With CRC}

The relationship between median serum ApoA-I levels and clinical variables in $250 \mathrm{CRC}$ patients is shown in Table 3 . In the entire cohort, ApoA-I levels were not associated with age, tobacco history, $\mathrm{pN}$ classification, pTNM stage, TG levels or ApoB levels. ApoA-I levels were significantly higher in women than in men $(p=0.001)$ and were higher in patients who did not have a history of alcohol use than in those who did $(p=0.017)$. However, the presence of metastasis $(p=0.010)$ and higher T classification $(p<0.001)$ were associated with decreased ApoA-I levels. Furthermore, in CRC patients, increased median ApoA-I levels were significantly associated with higher serum levels of HDL-C $(p=0.001)$ and TC $(p=0.001)$. The $\mathrm{CRC}$ patients with higher CRP levels $(p<0.001)$ displayed significantly decreased median ApoA-I levels. (Table 3)

In the entire cohort, 126 patients displayed lower ApoA-I levels $(<1.08 \mathrm{mg} / \mathrm{dL})$, and 124 patients displayed higher ApoA-I levels ( $\geq 1.08 \mathrm{mg} / \mathrm{dL})$. The distribution of the clinical characteristics of the included patients in the two groups stratified according to ApoA-I level are also presented in Table 3. The chi-square test showed that differences in gender $(p=0.001)$, alcohol history $(p=0.025)$, pT classification $(p=0.001), \mathrm{pM}$ classification $(p=0.002), \mathrm{pTNM}$ stage $(\mathrm{p}=0.023)$, CRP levels $(p=0.001)$, HDL-C levels $(p<0.001)$, LDL-C levels $(p=0.043)$, and TC levels ( $p=0.002)$ between the two groups were statistically significant. In contrast, age, tobacco history, $\mathrm{pN}$ classification, and TG levels were similar between the two groups.

\section{A Novel Prognostic Stratification System Based On The Combination Of ApoA-I And CRP Levels}

Because ApoA-I and CRP were independent risk factors for patients with CRC in this analysis, combining ApoA-I and CRP concentrations produced a novel prognostic stratification system in which the differentiated subsets more accurately reflected the actual outcomes of patients with CRC. The patients were classified as follows: low risk (ApoA-I level $\geq 1.08 \mathrm{mg} / \mathrm{dL}$ and CRP level $<3.04 \mathrm{mg} / \mathrm{dL}$ ), medium risk (ApoA-I level $\geq 1.08 \mathrm{mg} / \mathrm{dL}$ or CRP level $<3.04 \mathrm{mg} / \mathrm{dL}$ ), or high risk (ApoA-I level $<1.08 \mathrm{mg} /$ $\mathrm{dL}$ and CRP level $\geq 3.04 \mathrm{mg} / \mathrm{dL}$ ). In the entire CRC cohort, patient overall survival and disease-free survival differed significantly according to group (Figure $3 \mathrm{~A} ; p=0.001$, 
Table 2 Univariate And Multivariate Cox Hazards Analysis For Overall Survival In Patients With CRC

\begin{tabular}{|c|c|c|c|c|c|c|}
\hline \multirow[t]{2}{*}{ Variables } & \multicolumn{3}{|c|}{ Univariate Analysis } & \multicolumn{3}{|c|}{ Multivariate Analysis } \\
\hline & HR & $95 \% \mathrm{Cl}$ & $P$-value* & HR & $95 \% \mathrm{Cl}$ & $P$-value $* * *$ \\
\hline $\begin{array}{l}\text { Gender } \\
\text { Male vs. Female }\end{array}$ & 0.84 & $0.53-1.35$ & 0.479 & & & \\
\hline $\begin{array}{l}\text { Age(years) } \\
<58 \text { vs. } \geq 58\end{array}$ & 1.00 & $0.63-1.60$ & 0.996 & & & \\
\hline $\begin{array}{l}\text { Tobacco history } \\
\text { Yes vs. No }\end{array}$ & 1.12 & $0.67-1.86$ & 0.673 & & & \\
\hline $\begin{array}{c}\text { Alcohol history } \\
\text { Yes vs. No }\end{array}$ & 1.11 & $0.57-2.17$ & 0.757 & & & \\
\hline $\begin{array}{c}\text { PT classification } \\
\text { T4 vs. TI-3 }\end{array}$ & 1.76 & $1.08-2.86$ & 0.023 & & & \\
\hline $\begin{array}{c}\text { pN classification } \\
\text { NI-N3 vs. N0 }\end{array}$ & 3.48 & $1.97-6.15$ & $<0.001$ & & & \\
\hline $\begin{array}{l}\text { pMstatus } \\
\text { Yes vs. No }\end{array}$ & 6.46 & $3.90-10.70$ & $<0.001$ & & & \\
\hline $\begin{array}{l}\text { PTNM stage } \\
\text { IvsIlvs III vs IV }\end{array}$ & $\begin{array}{l}\& \\
2.56\end{array}$ & I.89-3.48 & $<0.001$ & 2.53 & $1.85-3.45$ & $<0.001$ \\
\hline $\begin{array}{c}\text { ApoA-I (mg/dL) } \\
<1.08 \text { vs. } \geq 1.08\end{array}$ & 0.47 & $0.28-0.76$ & 0.002 & 1.52 & $0.58-2.08$ & 0.023 \\
\hline $\begin{array}{l}\text { CRP }(\mathbf{m g} / \mathrm{dL}) \\
<3.04 \text { vs. } \geq 3.04\end{array}$ & 1.95 & $1.20-3.17$ & 0.007 & 1.85 & $1.16-2.26$ & 0.035 \\
\hline $\begin{array}{c}\text { HDL-C (mg/dL) } \\
<1.18 \text { vs. } \geq 1.18\end{array}$ & 0.50 & $0.3 \mathrm{I}-0.8 \mathrm{I}$ & 0.005 & 0.57 & $0.29-1.13$ & 0.43 \\
\hline $\begin{array}{l}\text { LDL-C (mg/dL) } \\
\quad<3.18 \text { vs. } \geq 3.18\end{array}$ & 1.01 & $0.63-1.62$ & 0.960 & & & \\
\hline $\begin{array}{l}\text { TC }(\mathbf{m g} / \mathbf{d L}) \\
\quad<5.08 \text { vs. } \geq 5.08\end{array}$ & 0.92 & $0.58-1.47$ & 0.734 & & & \\
\hline $\begin{array}{l}\text { TG (mg/dL) } \\
\quad<1.29 \text { vs. } \geq 1.29\end{array}$ & 0.82 & $0.5|-| .31$ & 0.405 & & & \\
\hline $\begin{array}{c}\text { ApoB (mg/dL) } \\
<0.97 \text { vs. } \geq 0.97\end{array}$ & 1.21 & $0.75-1.94$ & 0.434 & & & \\
\hline
\end{tabular}

Notes: $95 \% \mathrm{Cl}, 95 \%$ confidence interval. *Univariate analysis, $p<0.05$ considered as statistically significant. $* *$ Cox hazard regression model, $p<0.05$ considered as statistically significant. \&TNM denoted tumor-node-metastasis. Bold values indicate significant differences $(p<0.05)$

Abbreviation: HR, Hazard ratio.

Figure $3 \mathrm{~B} ; p=0.004$, respectively). The mean overall survival durations of the low-, medium-, and high-risk groups were 83.2 months (95\% CI: 77.2-89.2; $\mathrm{n}=75), 61.8$ months (95\% CI: 56.5-67.1; n=99), and 52.6 months (95\% CI: 45.3-59.9; $\mathrm{n}=76$ ), respectively. We also analyzed the prognostic value of this prognostic stratification system in selected CRC patient subgroups stratified according to TNM stage. In TNM stage I, II and III populations, the overall and disease-free survival durations of the CRC patients were not different between the three groups (Figure 3C-H). The overall survival was significantly different between the three groups of patients classified as TNM stage IV ( $\mathrm{n}=58, p=0.01$, Figure $3 \mathrm{I})$. Furthermore, for TNM stage IV CRC patients, the disease-free survival duration was also significantly different between the three groups $(\mathrm{n}=58, p=0.041$, Figure $3 \mathrm{~J})$. 

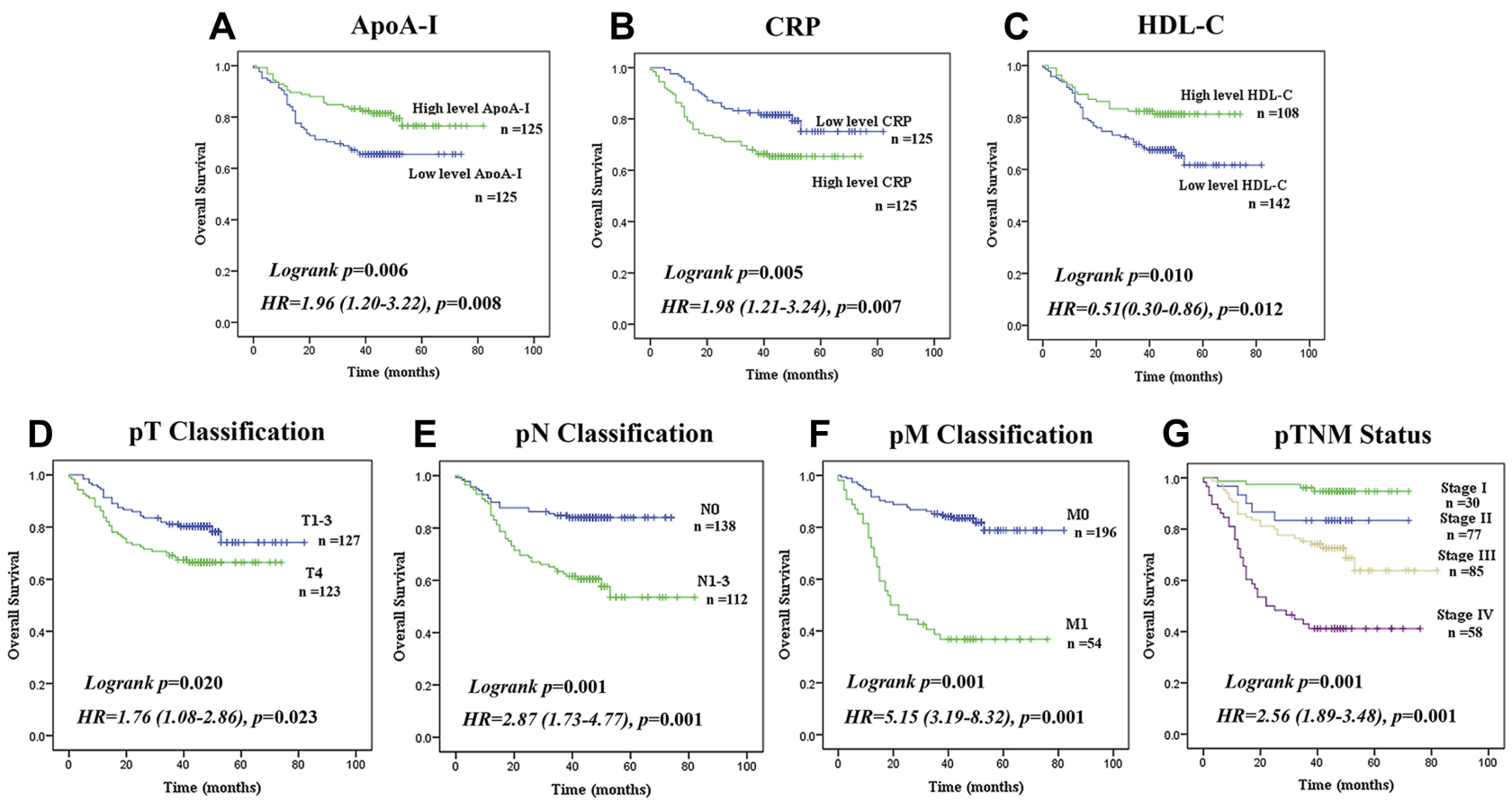

Figure I Analysis of overall survival in CRC patients. Kaplan-Meier survival curves for overall survival of CRC patients, according to serum level of (A) ApoA-I (Low level: $<$ I.08(mg/dL), n=125; High level: $\geq 1.08(\mathrm{mg} / \mathrm{dL}), \mathrm{n}=125)$, (B) CRP (Low level: $<3.04(\mathrm{mg} / \mathrm{dL}), \mathrm{n}=125$; High level: $\geq 3.04(\mathrm{mg} / \mathrm{dL}), \mathrm{n}=125),(\mathbf{C}) \mathrm{HDL}-\mathrm{C}(\mathrm{Low}$ level:< I.18(mg/dL), $\mathrm{n}=142$; High level: $\geq 1.18(\mathrm{mg} / \mathrm{dL}), \mathrm{n}=108)$ and classification of $(\mathbf{D}-\mathbf{G}) \mathrm{pT}, \mathrm{pN}, \mathrm{pM}$, and TNM stage. (log rank test used to calculate $P$-values.).

A

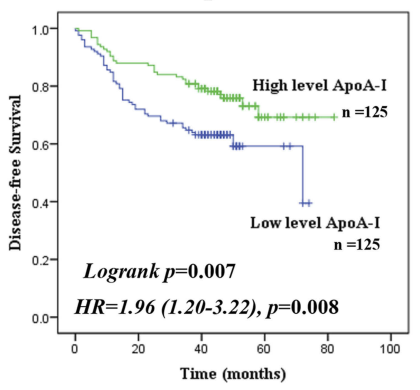

B

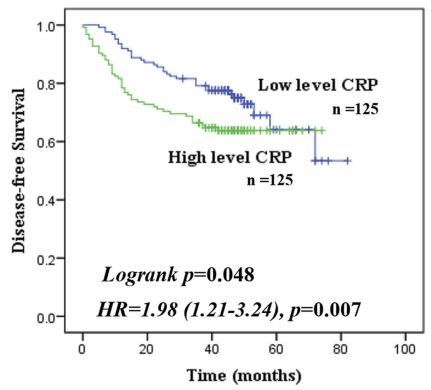

C HDL-C

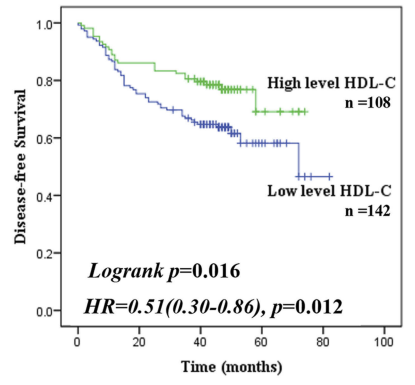

D pT classification

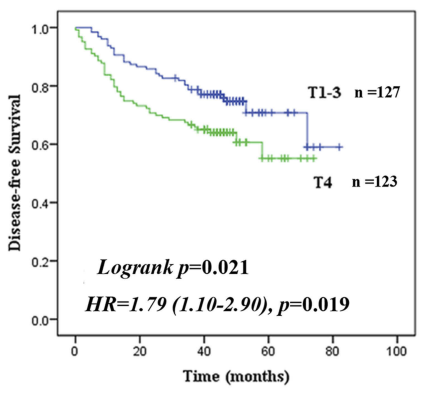

E pN classification

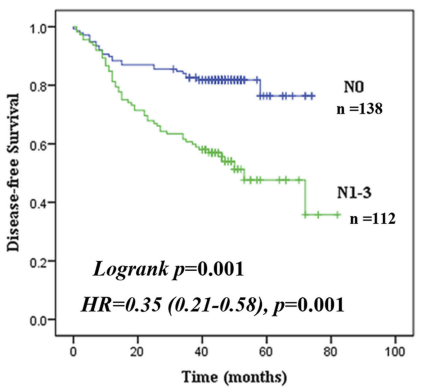

F $\quad$ pM classification

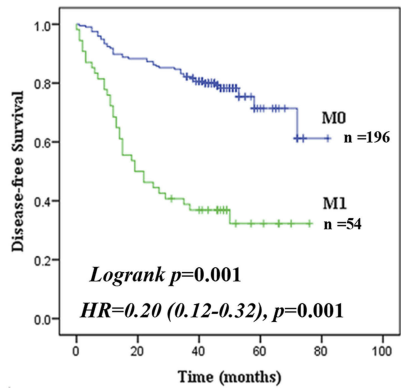

G

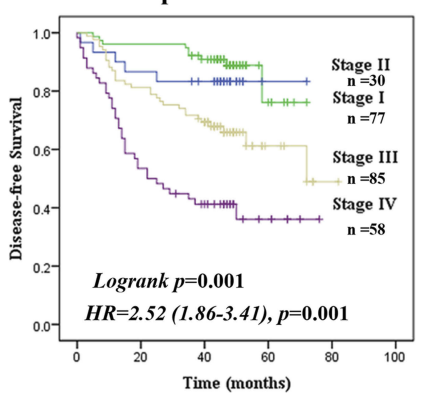

Figure 2 Analysis of disease-free survival in CRC patients. Disease-free survival curves of CRC patients, according to serum level of (A) ApoA-I (Low level: $<1.08(\mathrm{mg} / \mathrm{dL})$, $\mathrm{n}=125$; High level: $\geq 1.08(\mathrm{mg} / \mathrm{dL}), \mathrm{n}=125)$, (B) CRP (Low level:<3.04(mg/dL), n=125; High level: $\geq 3.04(\mathrm{mg} / \mathrm{dL}), \mathrm{n}=125)$, (C) HDL-C (Low level:< I. 18(mg/dL), $\mathrm{n}=142 ;$ High level: $\geq 1.18(\mathrm{mg} / \mathrm{dL}), \mathrm{n}=108)$ and classification of $(\mathrm{D}-\mathbf{G}) \mathrm{pT}$, pN, pM, and TNM stage. (log rank test used to calculate $P$-values.). 
Table 3 Relationship Between The ApoA-I Concentration And The Clinical Characteristics In Patients With CRC

\begin{tabular}{|c|c|c|c|c|c|c|}
\hline \multirow[t]{2}{*}{ Variables } & Case & $\begin{array}{l}\text { ApoA-I } \\
\text { (mg/dL) }\end{array}$ & \multirow[t]{2}{*}{ P-value* } & \multirow{2}{*}{$\begin{array}{l}\text { ApoA-I } \\
<\mathrm{I.08}(\mathrm{mg} / \mathrm{dL}) \\
\text { (n) }\end{array}$} & \multirow{2}{*}{$\begin{array}{l}\text { Apo-I } \\
\geq I .08(\mathrm{mg} / \mathrm{dL}) \\
\text { (n) }\end{array}$} & \multirow[t]{2}{*}{$P$-value $* *$} \\
\hline & (n) & (Median+range) & & & & \\
\hline \multicolumn{7}{|l|}{ Age(years) } \\
\hline$<58$ & 119 & $1.04(0.46-1.85)$ & 0.290 & 66 & 53 & 0.127 \\
\hline$\geq 58$ & 131 & I.I (0.43-2.56) & & 60 & 71 & \\
\hline \multicolumn{7}{|l|}{ Gender } \\
\hline Male & 144 & $1.03(0.43-2.56)$ & 0.001 & 86 & 58 & 0.001 \\
\hline Female & 106 & I. $14(0.55-2.01)$ & & 40 & 66 & \\
\hline \multicolumn{7}{|l|}{ Tobacco history } \\
\hline No & 180 & $1.07(0.46-2.01)$ & 0.078 & 87 & 93 & 0.295 \\
\hline Yes & 70 & $1.04(0.43-2.56)$ & & 39 & 31 & \\
\hline \multicolumn{7}{|l|}{ Alcohol history } \\
\hline No & 217 & $1.08(0.43-2.56)$ & 0.017 & 103 & 114 & 0.025 \\
\hline Yes & 32 & $1.01(0.69-1.36)$ & & 22 & 10 & \\
\hline TI-T3 & 40 & $1.13(0.43-2.56)$ & 0.001 & 48 & 79 & 0.001 \\
\hline \multicolumn{7}{|l|}{ pT classification } \\
\hline $\mathrm{T} 4$ & 186 & $1.04(0.46-1.85)$ & & 77 & 46 & \\
\hline \multicolumn{7}{|l|}{ pN classification } \\
\hline No & 116 & $1.08(0.60-2.01)$ & 0.735 & 57 & 59 & 0.342 \\
\hline $\mathrm{NI}-\mathrm{N} 3$ & 110 & $1.06(0.46-2.56)$ & & 61 & 49 & \\
\hline \multicolumn{7}{|l|}{ pMetastasis } \\
\hline No & 173 & I.09(0.5I-2.56) & 0.010 & 80 & 93 & 0.002 \\
\hline Yes & 54 & $1.00(0.46-1.85)$ & & 38 & 16 & \\
\hline pTNM stage & $\&$ & & & & & \\
\hline I & 30 & $1.11(0.43-1.75)$ & 0.116 & 14 & 16 & 0.023 \\
\hline II & 77 & $1.13(0.09-2.01)$ & & 32 & 45 & \\
\hline III & 85 & $1.09(0.5 I-2.56)$ & & 40 & 45 & \\
\hline IV & 58 & $1.03(0.46-1.85)$ & & 39 & 19 & \\
\hline \multicolumn{7}{|l|}{ CRP (mg/dL) } \\
\hline$<3.04$ & 125 & $1.16(0.43-1.86)$ & $<0.001$ & 50 & 75 & 0.001 \\
\hline$\geq 3.04$ & 125 & $1.05(0.46-2.56)$ & & 76 & 49 & \\
\hline \multicolumn{7}{|l|}{ HDL-C (mg/dL) } \\
\hline$<1.18$ & 142 & $0.94(0.46-1.85)$ & $<0.001$ & 112 & 30 & $<0.001$ \\
\hline$\geq 1.18$ & 108 & $1.26(0.43-2.56)$ & & 23 & 85 & \\
\hline \multicolumn{7}{|l|}{ LDL-C (mg/dL) } \\
\hline$<3.18$ & 125 & $1.08(0.46-1.65)$ & 0.018 & 71 & 54 & 0.043 \\
\hline$\geq 3.18$ & 125 & $1.14(0.43-2.56)$ & & 55 & 70 & \\
\hline \multicolumn{7}{|l|}{ TC (mg/dL) } \\
\hline$<5.08$ & 123 & $1.06(0.43-1.93)$ & 0.001 & 74 & 49 & 0.002 \\
\hline$\geq 5.08$ & 127 & I.15(0.46-2.56) & & 52 & 75 & \\
\hline \multicolumn{7}{|l|}{ TG (mg/dL) } \\
\hline$<1.29$ & 124 & I.12(0.43-2.56) & 0.220 & 56 & 68 & 0.100 \\
\hline$\geq 1.29$ & 126 & $1.09(0.46-1.89)$ & & 70 & 56 & \\
\hline
\end{tabular}

(Continued) 
Table 3 (Continued).

\begin{tabular}{|c|c|c|c|c|c|c|}
\hline \multirow[t]{2}{*}{ Variables } & Case & $\begin{array}{l}\text { ApoA-I } \\
(\mathrm{mg} / \mathrm{dL})\end{array}$ & \multirow[t]{2}{*}{$P$-value* } & $\begin{array}{l}\text { ApoA-I } \\
<1.08(\mathrm{mg} / \mathrm{dL})\end{array}$ & $\begin{array}{l}\text { Apo-I } \\
\geq 1.08(\mathrm{mg} / \mathrm{dL})\end{array}$ & \multirow[t]{2}{*}{$P$-value** } \\
\hline & (n) & (Median+range) & & (n) & (n) & \\
\hline \multicolumn{7}{|l|}{ ApoB (mg/dL) } \\
\hline$<0.97$ & 119 & $1.11(0.43-2.56)$ & 0.964 & 61 & 58 & 0.795 \\
\hline$\geq 0.97$ & $|3|$ & I.II(0.24-I.98) & & 65 & 66 & \\
\hline
\end{tabular}

Notes: $* P$ values were calculated by the Mann-Whitney $U$-test or Kruskal-Wallis test, bold values indicate significant differences $(P<0.05)$. ${ }^{* * P}$ values were calculated by the chi-square test $\left(\chi^{2}\right.$ test), $P<0.05$ considered as statistically significant. \&TNM denoted tumor-node-metastasis.

\section{Discussion}

In the present study, the prognostic values of lipids, including ApoA-I, Apo-B, HDL-C, LDL-C, TC, TG and $\mathrm{CRP}$, in patients with $\mathrm{CRC}$ were evaluated. Based on univariate Cox regression analyses, $\mathrm{pT}$ status, $\mathrm{pN}$ status, pM status, pTNM stage, and serum levels of ApoA-I, HDL-C and CRP were associated with the overall survival of CRC patients. Additionally, multivariate analysis

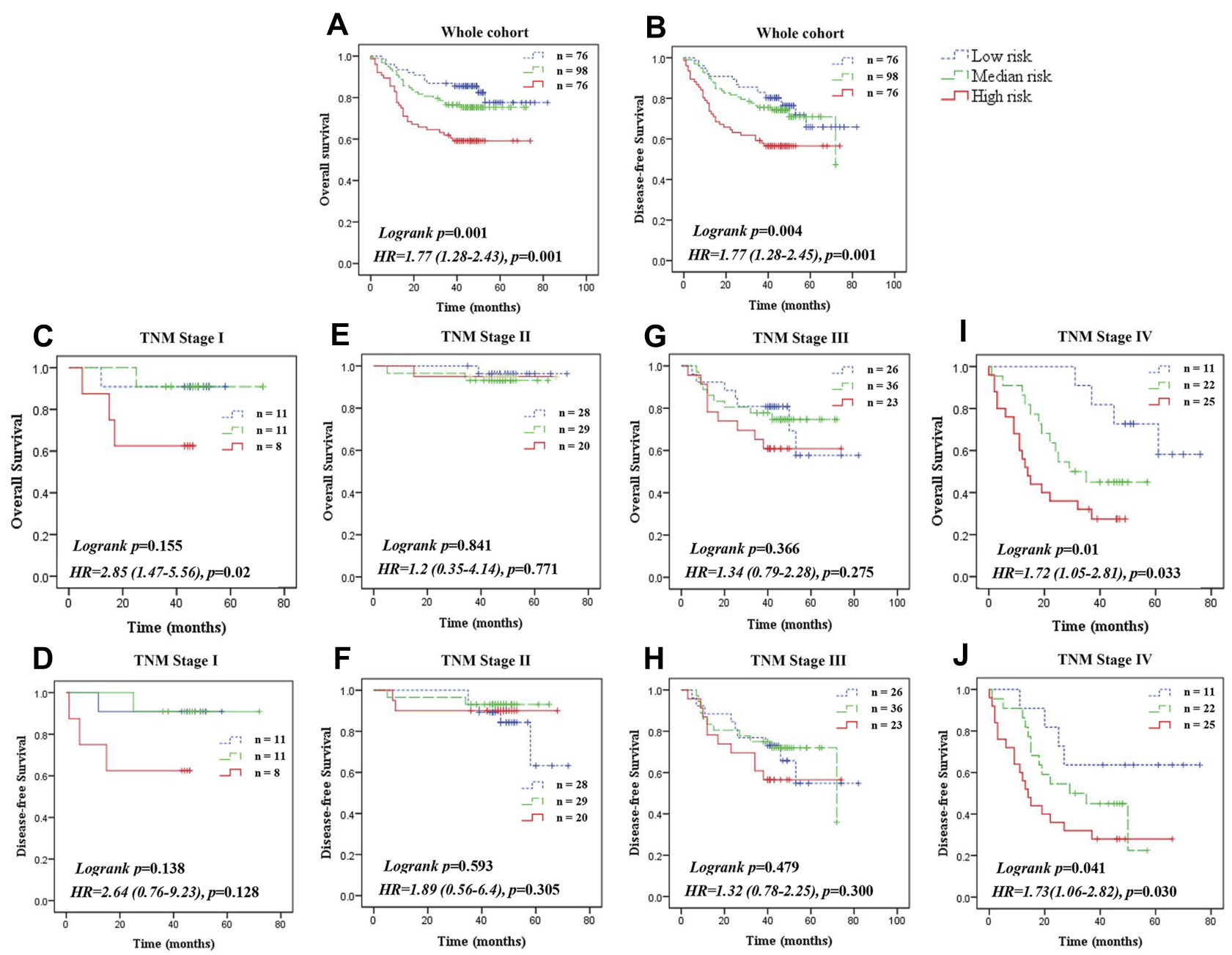

Figure 3 Three-tiered stratification of patients with colorectal cancer, incorporating two independent prognostic variables (ApoA-I and CRP concentrations). Three-tiered are grouped as follows: low-risk (ApoA-I $\geq 1.08 \mathrm{mg} / \mathrm{dL}$ and CRP $<3.04 \mathrm{mg} / \mathrm{dL}$ ); medium-risk (ApoA-l $\geq 1.08 \mathrm{mg} / \mathrm{dL}$ or $\mathrm{CRP}<3.04 \mathrm{mg} / \mathrm{dL}$ ); high-risk (ApoA-I $<1.08 \mathrm{mg} / \mathrm{dL}$ and CRP $\geq 3.04 \mathrm{mg} / \mathrm{dL}$ ). (A) Overall survival of patients in the three-tiered groups of the whole cohort. (B) Disease-free survival of patients in the three-tiered groups of the whole cohort. (C, D) Overall survival and disease-free survival of patients in the three-tiered groups of the TNM stage I cohort. (E, F) Overall survival and disease-free survival of patients in the three-tiered groups of the TNM stage Il cohort. (G, H) Overall survival and disease-free survival of patients in the three-tiered groups of the TNM stage III cohort. (I, J) Overall survival and disease-free survival of patients in the three-tiered groups of the TNM stage IV cohort. (The Kaplan-Meier method using the logrank test was used to calculate $P$-values, and the Cox proportional hazard regression model was employed to determine hazard ratio estimates). 
showed that pTNM stage, ApoA-I levels and CRP levels were independent prognostic indicators of CRC patient survival. Moreover, based on Kaplan-Meier survival analysis, higher levels of ApoA-I or HDL-C were significantly predictive of longer overall survival, whereas higher levels of CRP predicted poor overall survival. Therefore, ApoA-I and CRP before therapy may serve as novel independent prognostic factors for CRC. After adjustment for clinical characteristics, the ApoA-I level was associated with gender, alcohol history, pT classification, $\mathrm{pM}$ status, TNM stage and serum levels of CRP, HDL-C, LDL-C and TC.

Because the detection of ApoA-I is simple and inexpensive, this parameter is widely used in clinical laboratories to reflect lipid metabolism. Therefore, using ApoA-I as a prognostic biomarker for overall survival among CRC patients is convenient and acceptable. However, the role of ApoA-I in carcinogenesis and CRC pathogenesis is complex and multifactorial, and the associated mechanisms are not entirely understood. This lack of knowledge limits the application of ApoA-I as a prognostic factor in clinical settings. Angiogenesis is a complicated process requiring the coordinated action of a variety of growth factors and cell adhesion molecules in endothelial and mural cells, and this process plays a critical role in the growth and metastasis of solid tumors. ${ }^{31}$ Vascular endothelial growth factor (VEGF) and basic fibroblast growth factor (bFGF) are critical proangiogenic factors involved in all phases of angiogenesis, including the growth, migration, and differentiation of endothelial cells. ${ }^{32}$ A two-fold to four-fold increase in VEGF expression leads to considerable tumorigenic competence in CRC. ${ }^{33}$ FengGao and Farias-Eisner showed that ApoA-I mimetic peptides reduced tumor angiogenesis ${ }^{34}$ and suppressed tumor growth and development in mouse models of ovarian cancer. ${ }^{35}$ The results of these studies can better help clarify the mechanisms underlying the function of ApoA-I in tumor prevention. Lysophospholipids, such as lysophosphatidic acid (LPA), are well-known activators of proliferation in many cancer types. LPA from the circulation or produced by tumors binds to LPA receptors and stimulates the production and secretion of proangiogenic factors, including VEGF. VEGF binds to VEGFR2, activates downstream signaling molecules, and induces angiogenesis. One plausible mechanism for the antitumor activity of ApoA-I is that ApoA-I inhibits LPA-induced VEGF production, thus blocking the VEGF-mediated activation of VEGFR2 and downstream signaling pathways, consequently suppressing tumor angiogenesis. In addition, Zamanian-Daryoush et al demonstrated that ApoA-I may exert antitumor effects indirectly by altering the function of macrophages and modulating the basic elements of both innate and adaptive immunity. ${ }^{23}$ Our results showed that patients with lower ApoA-I levels had significantly poorer overall survival, and this finding is consistent with the results of these previous studies. However, the mechanisms underlying the function of ApoA-I must be further explored.

Inflammation is a hallmark of cancer, and many studies have indicated that chronic inflammation is associated with an increased risk of developing CRC. ${ }^{36-39} \mathrm{CRC}$ is a wellrecognized marker of inflammation. ${ }^{40}$ Ishizuka demonstrated that an increased CRP concentration was a strong independent prognostic factor of CRC. ${ }^{41}$ Our results showed that CRC patients with higher CRP levels also had poorer overall survival. Additionally, the level of CRP was associated with pT status and pM status, as well as the ApoA-I level. In addition to its known functions, ApoA-I also possesses anti-inflammatory and antioxidant properties. ApoA-I mimetic peptides inhibit inflammatory responses via their remarkable ability to bind to and remove bioactive lipids, such as LPA, from the circulation. ${ }^{42,43}$

Moreover, patients with moderate or advanced disease exhibited lower ApoA-I levels and higher CRP levels than patients with early-stage disease, and this more advanced disease stage led to shorter overall survival. Considering the opposing effects of ApoA-I and CRP on CRC development, the combination of ApoA-I and CRP concentrations served as a novel prognostic stratification system that separated patients with CRC into three significantly distinguished risk groups that more accurately reflected the actual outcomes of CRC patients. In the entire CRC cohort, patient overall survival and diseasefree survival differed significantly between the low-risk, medium-risk and high-risk groups. Additionally, the prognostic grouping system was effective in stratifying the selected patient populations. The overall survival and disease-free survival were significantly diverse between the low-risk, mediumrisk and high-risk groups in CRC patients who were classified as TNM stage IV. However, there were no significant differences between the three groups in terms of TNM stage I, stage II and stage III. The low-risk and medium-risk groups showed better prospective overall survival than did the high-risk group among patients with advanced disease.

Although ApoA-I was reported to be a prognostic factor in several malignancies, this study is the first to combine Apo-I with CRP as a novel prognostic stratification system for the overall survival of colorectal cancer 
patients. Although our study is based on a relatively large sample size with a long follow-up period, it has some limitations. Similar to all other retrospective studies, our study is inevitably subject to multiple biases.

In summary, we analyzed the clinical prognostic significance of preoperative lipid profiles and CRP levels in $250 \mathrm{CRC}$ patients. In this study, ApoA-I and CRP were independent prognostic factors for CRC in both the entire cohort and subpopulations stratified according to $\mathrm{T}$ classification, $\mathrm{N}$ classification and metastasis status. Prognostic markers for CRC are currently lacking, and ApoA-I and CRP levels can be measured using a highly reproducible assay that can easily be conducted in all diagnostic laboratories. Thus, the combination of ApoA-I and CRP may serve as a novel prognostic stratification system for the overall survival of colorectal cancer patients.

\section{Abbreviations}

ApoA-I, apolipoprotein A-I; CRC, colorectal cancer; HDL$\mathrm{C}$, high-density lipoprotein-cholesterol; CRP, C-reactive protein; Apo-B, apolipoprotein B; TC, total cholesterol; LDL-C, low-density lipoprotein-cholesterol; TG, triglyceride; VEGF, vascular endothelial growth factor; b-FGF, basic fibroblast growth factor; LPA, lysophosphatidic acid.

\section{Availability Of Data And Materials}

Due to ethical restrictions, the raw data for this paper are available upon request from the corresponding author.

\section{Acknowledgment}

We thank the staff of the Biochemical Lab of Sun Yat-Sen University Cancer Center who provided various biochemical markers and all the staff who supported our study.

\section{Author Contributions}

JY and QL carried out the main work and contributed equally. They participated in the conception, design, acquisition of data and drafted the manuscript. XW, ZL and MC performed interpretation of data and statistical analysis. $\mathrm{LZ}$ and $\mathrm{HH}$, conceived the study, participated in its design and coordination and confirmed the final approval of the version to be published. All authors contributed to data analysis, drafting or revising the article, gave final approval of the version to be published, and agree to be accountable for all aspects of the work.

\section{Funding}

This paper is supported by grant from the Natural Science Foundation of Guangdong Province China (grant no. 2018A030310260).

\section{Disclosure}

The authors report no conflicts of interest in this work.

\section{References}

1. Ferlay J, Shin H-R, Bray F, Forman D, Mathers C, Parkin DM. Estimates of worldwide burden of cancer in 2008: GLOBOCAN 2008. Int J Cancer. 2010;127(12):2893-2917. doi:10.1002/ijc.25516

2. Ishizuka M, Nagata H, Takagi K, Iwasaki Y, Shibuya N, Kubota K. Clinical significance of the C-reactive protein to albumin ratio for survival after surgery for colorectal cancer. Ann Surg Oncol. 2016;23 (3):900-907. doi:10.1245/s10434-015-4948-7

3. Seong MK. Prognostic inflammation score in surgical patients with colorectal cancer. J Korean Med Sci. 2015;30(12):1793-1799. doi:10.3346/jkms.2015.30.12.1793

4. Jung YS, Ryu S, Chang Y, et al. Associations between parameters of glucose and lipid metabolism and risk of colorectal neoplasm. Dig Dis Sci. 2015;60(10):2996-3004. doi:10.1007/s10620-015-3713-x

5. Luo X, Cheng C, Tan Z, et al. Emerging roles of lipid metabolism in cancer metastasis. Mol Cancer. 2017;16(1):76. doi:10.1186/s12943-0170646-3

6. Chang SJ, Hou M-F, Tsai S-M, et al. The association between lipid profiles and breast cancer among Taiwanese women. Clin Chem Lab Med. 2007;45(9):1219-1223. doi:10.1515/CCLM.2007.263

7. Zhang X, Zhao X-W, Liu D-B, et al. Lipid levels in serum and cancerous tissues of colorectal cancer patients. World J Gastroenterol. 2014;20 (26):8646-8652. doi:10.3748/wjg.v20.i26.8646

8. Cruz PM, Mo H, McConathy WJ, Sabnis N, Lacko AG. The role of cholesterol metabolism and cholesterol transport in carcinogenesis: a review of scientific findings, relevant to future cancer therapeutics. Front Pharmacol. 2013;4:119. doi:10.3389/fphar.2013.00119

9. Long J, Zhang C-J, Zhu N, et al. Lipid metabolism and carcinogenesis, cancer development. Am J Cancer Res. 2018;8(5):778-791.

10. Jiang R, Yang ZH, Luo DH, et al. Elevated apolipoprotein A-I levels are associated with favorable prognosis in metastatic nasopharyngeal carcinoma. Med Oncol. 2014;31(8):80. doi:10.1007/s12032-014-0374-0

11. Zhang Z, Bast RC, Yu Y, et al. Three biomarkers identified from serum proteomic analysis for the detection of early stage ovarian cancer. Cancer Res. 2004;64(16):5882-5890. doi:10.1158/0008-5472.CAN-04-0746

12. Ehmann M, Felix K, Hartmann D, et al. Identification of potential markers for the detection of pancreatic cancer through comparative serum protein expression profiling. Pancreas. 2007;34(2):205-214. doi:10.1097/01.mpa.0000250128.57026.b2

13. Frank PG, Marcel YL. Apolipoprotein A-I: structure-function relationships. J Lipid Res. 2000;41(6):853-872.

14. Sorci-Thomas MG, Bhat S, Thomas MJ. Activation of lecithin: cholesterolacyltransferase by HDL ApoA-I central helices. Clin Lipidol. 2009;4(1):113-124. doi:10.2217/17584299.4.1.113

15. Huang HL, Stasyk T, Morandell S, et al. Biomarker discovery in breast cancer serum using 2-D differential gel electrophoresis/ MALDI-TOF/TOF and data validation by routine clinical assays. Electrophoresis. 2006;27(8):1641-1650. doi:10.1002/elps.200500857

16. Sung KC, Ryu S, Wild SH, Byrne CD. An increased high-density lipoprotein cholesterol/apolipoprotein A-I ratio is associated with increased cardiovascular and all-cause mortality. Heart. 2015;101 (7):553-558. doi:10.1136/heartjnl-2014-306784 
17. de Souza JA, Vindis C, Nègre-Salvayre A, et al. Small, dense HDL 3 particles attenuate apoptosis in endothelial cells: pivotal role of apolipoprotein A-I. $J$ Cell Mol Med. 2010;14(3):608-620. doi: $10.1111 /$ j. $1582-4934.2009 .00713 . x$

18. Fuhrman B, Gantman A, Aviram M. Paraoxonase 1 (PON1) deficiency in mice is associated with reduced expression of macrophage SR-BI and consequently the loss of HDL cytoprotection against apoptosis. Atherosclerosis. 2010;211(1):61-68. doi:10.1016/j. atherosclerosis.2010.01.025

19. Bhattacharyya T, Nicholls SJ, Topol EJ, et al. Relationship of paraoxonase 1 (PON1) gene polymorphisms and functional activity with systemic oxidative stress and cardiovascular risk. JAMA. 2008;299 (11):1265-1276. doi:10.1001/jama.299.11.1265

20. Lu H, Ouyang W, Huang C. Inflammation, a key event in cancer development. Mol Cancer Res. 2006;4(4):221-233. doi:10.1158/ 1541-7786.MCR-05-0261

21. Kobayashi Y, Kashima H, Wu R-C, et al. Mevalonate pathway antagonist suppresses formation of serous tubal intraepithelial carcinoma and ovarian carcinoma in mouse models. Clin Cancer Res. 2015;21(20):4652-4662. doi:10.1158/1078-0432.CCR-14-3368

22. Coussens LM, Zitvogel L, Palucka AK. Neutralizing tumor-promoting chronic inflammation: a magic bullet? Science. 2013;339 (6117):286-291. doi:10.1126/science.1232227

23. Zamanian-Daryoush M, Lindner D, Tallant TC, et al. The cardioprotective protein apolipoprotein A1 promotes potent anti-tumorigenic effects. J Biol Chem. 2013;288(29):21237-21252. doi:10.1074/jbc. M113.468967

24. Kim YW, Bae SM, Lim H, et al. Development of multiplexed beadbased immunoassays for the detection of early stage ovarian cancer using a combination of serum biomarkers. PLOS ONE. 2012;7(9): e44960. doi:10.1371/journal.pone. 0050900

25. Tuft Stavnes H, Nymoen DA, Hetland Falkenthal TE, Kærn J, Tropé CG, Davidson B. APOA1 mRNA expression in ovarian serous carcinoma effusions is a marker of longer survival. Am J Clin Pathol. 2014;142(1):51-57. doi:10.1309/AJCPD8NBSHXRXQL7

26. Luo XL, Zhong G-Z, Hu L-Y, et al. Serum apolipoprotein A-I is a novel prognostic indicator for non-metastatic nasopharyngeal carcinoma. Oncotarget. 2015;6(41):44037-44048. doi:10.18632/oncotarget.5823

27. van Duijnhoven FJ, Bueno-De-Mesquita HB, Calligaro M, et al. Blood lipid and lipoprotein concentrations and colorectal cancer risk in the European prospective investigation into cancer and nutrition. Gut. 2011;60(8):1094-1102. doi:10.1136/gut.2010.225011

28. Gelfo V, Rodia MT, Pucci M, et al. A module of inflammatory cytokines defines resistance of colorectal cancer to EGFR inhibitors. Oncotarget. 2016;7(44):72167-72183. doi:10.18632/oncotarget.12354

29. Li C, Xu Q, Chen L, Luo C, Ying J, Liu J. C-reactive protein (CRP) as a prognostic factor for colorectal cancer after surgical resection of pulmonary metastases. Bull Cancer. 2017;104(3):232-236. doi:10.1016/j.bulcan.2016.11.016

30. Gelfo V, Mazzeschi M, Grilli G, et al. A novel role for the interleukin-1 receptor axis in resistance to anti-EGFR therapy. Cancers (Basel). 2018;10(10). doi:10.3390/cancers10110400.
31. Welti J, Loges S, Dimmeler S, Carmeliet P. Recent molecular discoveries in angiogenesis and antiangiogenic therapies in cancer. $J$ Clin Invest. 2013;123(8):3190-3200. doi:10.1172/JCI70212

32. Hu T, Li LF, Shen J, Zhang L, Cho CH. Chronic inflammation and colorectal cancer: the role of vascular endothelial growth factor. Curr Pharm Des. 2015;21(21):2960-2967. doi:10.2174/1381612821666 150514104244

33. Okada F, Rak JW, Croix BS, et al. Impact of oncogenes in tumor angiogenesis: mutant $\mathrm{K}$-ras up-regulation of vascular endothelial growth factor/vascular permeability factor is necessary, but not sufficient for tumorigenicity of human colorectal carcinoma cells. Proc Natl Acad Sci U S A. 1998;95(7):3609-3614. doi:10.1073/ pnas.95.7.3609

34. Gao F, Vasquez SX, Su F, et al. L-5F, an apolipoprotein A-I mimetic, inhibits tumor angiogenesis by suppressing VEGF/basic FGF signaling pathways. Integr Biol (Camb). 2011;3(4):479-489. doi:10.1039/ c0ib00147c

35. Su F, Kozak KR, Imaizumi S, et al. Apolipoprotein A-I (apoA-I) and apoA-I mimetic peptides inhibit tumor development in a mouse model of ovarian cancer. Proc Natl Acad Sci U S A. 2010;107 (46):19997-20002. doi:10.1073/pnas.1009010107

36. Lasry A, Zinger A, Ben-Neriah Y. Inflammatory networks underlying colorectal cancer. Nat Immunol. 2016;17(3):230-240. doi:10.1038/ ni. 3384

37. Garcia-Anguita A, Kakourou A, Tsilidis KK. Biomarkers of inflammation and immune function and risk of colorectal cancer. Curr Colorectal Cancer Rep. 2015;11(5):250-258. doi:10.1007/s11888015-0282-5

38. Romano M, DE Francesco F, Zarantonello L, et al. From inflammation to cancer in inflammatory bowel disease: molecular perspectives. Anticancer Res. 2016;36(4):1447-1460.

39. Erdogan S, Yilmaz FM, Yazici O, et al. Inflammation and chemerin in colorectal cancer. Tumour Biol. 2016;37(5):6337-6342. doi:10.1007/s13277-015-4483-y

40. Cahlin C, Lönnroth C, Arvidsson A, Nordgren S, Lundholm K. Growth associated proteins in tumor cells and stroma related to disease progression of colon cancer accounting for tumor tissue PGE2 content. Int J Oncol. 2008;32(4):909-918.

41. Ishizuka M, Nagata H, Takagi K, Horie T, Kubota K. Inflammationbased prognostic score is a novel predictor of postoperative outcome in patients with colorectal cancer. Ann Surg. 2007;246(6):1047-1051. doi:10.1097/SLA.0b013e3181454171

42. Navab M, Anantharamaiah GM, Reddy ST, et al. Apolipoprotein A-I mimetic peptides. Arterioscler Thromb Vasc Biol. 2005;25(7):13251331. doi:10.1161/01.ATV.0000165694.39518.95

43. Van Lenten BJ, Wagner AC, Jung C-L, et al. Anti-inflammatory apoA-I-mimetic peptides bind oxidized lipids with much higher affinity than human apoA-I. J Lipid Res. 2008;49(11):2302-2311. doi:10.1194/j1r.M800075-JLR200

\section{Publish your work in this journal}

Cancer Management and Research is an international, peer-reviewed open access journal focusing on cancer research and the optimal use of preventative and integrated treatment interventions to achieve improved outcomes, enhanced survival and quality of life for the cancer patient.
The manuscript management system is completely online and includes a very quick and fair peer-review system, which is all easy to use. Visit http://www.dovepress.com/testimonials.php to read real quotes from published authors. 